\title{
Research Article \\ Solitonic Solutions for Homogeneous KdV Systems by Homotopy Analysis Method
}

\author{
Mohammed Ali, Marwan Alquran, and Mahmoud Mohammad \\ Department of Mathematics and Statistics, Jordan University of Science and Technology, Irbid 22110, Jordan \\ Correspondence should be addressed to Mohammed Ali, myali@just.edu.jo
}

Received 14 July 2012; Accepted 28 August 2012

Academic Editor: J. C. Butcher

Copyright (C) 2012 Mohammed Ali et al. This is an open access article distributed under the Creative Commons Attribution License, which permits unrestricted use, distribution, and reproduction in any medium, provided the original work is properly cited.

We study two-component evolutionary systems of a homogeneous KdV equations of second and third order. The homotopy analysis method (HAM) is used for analytical treatment of these systems. The auxiliary parameter $h$ of HAM is freely chosen from the stability region of the $h$-curve obtained for each proposed system.

\section{Introduction}

Applications in physics are modeled by nonlinear systems. Very few nonlinear systems have closed form solutions, therefore, many researchers stress their goals to search numerical solutions. Homotopy analysis method (HAM), first proposed by Liao [1], is an elegant method which has proved its effectiveness and efficiency in solving many types of nonlinear equations [2,3]. Liao in his book [4] proved that HAM is a generalization of some previously used techniques such as the d-expansion method, artificial small parameter method [5], and Adomian decomposition method. Moreover, unlike previous analytic techniques, the HAM provides a convenient way to adjust and control the region and rate of convergence [6]. Recently, new interested applications of the homotopy analysis have been introduced by Abbasbandy and coauthors [7, 8]. Also, in [9] HAM is used to study the effects of thermocapillarity and thermal radiation on flow and heat transfer in a thin liquid film.

In this work, we consider a two-component evolutionary system of a homogeneous $\mathrm{KdV}$ equations of third order type (I) and (II) given, respectively, by

$$
\begin{gathered}
u_{t}=u_{x x x}+u u_{x}+v v_{x}, \\
v_{t}=-2 v_{x x x}-u v_{x}
\end{gathered}
$$




$$
\begin{gathered}
u_{t}=u_{x x x}+2 v u_{x}+u v_{x}, \\
v_{t}=u u_{x} .
\end{gathered}
$$

Also, we study a two-component evolutionary system of a homogeneous KdV equations of second order given by

$$
\begin{gathered}
u_{t}=-3 v_{x x}, \\
v_{t}=4 u_{x x}+u^{2} .
\end{gathered}
$$

In the literature many other direct mathematical methods such as the sine-cosine method, rational sine-cosine method, and extended tanh-coth method [10-14] have been implemented in obtaining different solitonic solutions to these systems. Our main goal in this paper is to see how much accuracy we may gain by applying HAM to such evolutionary systems.

In what follows, we highlight the main features of the homotopy analysis method. More details and examples can be found in $[7,8,15]$ and the references therein.

\section{Survey of Homotopy Analysis Method}

To illustrate the basic ideas of this method, we consider the following nonlinear system of differential equations:

$$
N_{j}\left[u_{1}(x, t), u_{2}(x, t), \cdots u_{m}(x, t)\right]=0, \quad j=1, \ldots, n,
$$

where $N_{j}$ are nonlinear operators, $t$ is an independent variable, $u_{i}(t)$ are unknown functions. By means of generalizing the traditional homotopy method, Liao construct the zeroth-order deformation equation as follows:

$$
(1-q) L_{j}\left[\phi_{i}(x, t, q)-u_{i, 0}(x, t)\right]=q h H(x, t) N_{j}\left[\phi_{1}(x, t, q), \ldots, \phi_{m}(x, t, q)\right],
$$

where $i=1, \ldots, m, j=1, \ldots, n, q \in[0,1]$ is an embedding parameter, $L_{j}$ is a general differential linear operator, $u_{i, 0}(x, t)$ are initial guesses of $u_{i}(x, t), \phi_{i}(x, t, q)$ are unknown functions, and $h$ and $H(x, t)$ are auxiliary parameter and auxiliary function, respectively. It is important note that, one has great freedom to choose auxiliary objects such as $h$ and $L_{j}$ in HAM; this freedom plays an important role in establishing the key stone of validity and flexibility of HAM as shown in this paper. obviously, when $q=0$ and $q=1$, both

$$
\phi_{i}(x, t, 0)=u_{i, 0}(x, t), \quad \phi_{i}(x, t, 1)=u_{i}(x, t), \quad i=1, \ldots, m,
$$

thus as $q$ increasing from 0 to 1 , the solutions of $\phi_{i}(x, t, q)$ change from the initial guesses $u_{i, 0}(x, t)$ to the solutions $u_{i}(x, t)$. Expanding $\phi_{i}(x, t, q)$ in Taylor series with respect to $q$, one has

$$
\phi_{i}(x, t, q)=u_{i, 0}(x, t)+\sum_{k=1}^{+\infty} u_{i, k}(x, t) q^{k}, \quad i=1, \ldots, m,
$$


where

$$
u_{i, k}(x, t)=\left.\frac{1}{k !} \frac{\partial^{k} \phi_{i}(x, t, q)}{\partial q^{k}}\right|_{q=0}, \quad i=1, \ldots, m
$$

if the auxiliary linear operators, the initial guesses, the auxiliary parameter $h$, and the auxiliary function are so properly chosen, then the series (2.4) converges at $q=1$, then one has

$$
\phi_{i}(x, t, 1)=u_{i, 0}(x, t)+\sum_{k=1}^{+\infty} u_{i, k}(x, t), \quad i=1, \ldots, m,
$$

which must be one of the solutions of the original nonlinear equations, as proved by liao. Define the vector

$$
\vec{u}_{i, n}(x, t)=\left(u_{i, 0}(x, t), u_{i, 1}(x, t), \ldots, u_{i, n}(x, t)\right), \quad i=1, \ldots, m
$$

Differentiating (2.2), $k$ times with respect to the embedding parameter $q$ and then setting $q=0$ and finally dividing them by $k$, we have the so-called $k$ th-order deformation equation

$$
L_{j}\left[u_{i, k}(x, t)-x_{k} u_{i, k-1}(x, t)\right]=h R_{j, k}\left(\vec{u}_{i, k-1}(x, t)\right), \quad i=1, \ldots, m, j=1, \ldots, n,
$$

subject to the initial conditions

$$
L_{j}(0)=0,
$$

where

$$
\begin{array}{r}
R_{j, k}\left(\vec{u}_{i, k-1}(x, t)\right)=\left.\frac{1}{(k-1) !} \frac{\partial^{k-1} N_{j}\left[\phi_{1}(x, t, q), \phi_{2}(x, t, q), \ldots, \phi_{m}(x, t, q)\right]}{\partial q^{k-1}}\right|_{q=0}, \\
x_{m}=0, \quad m \leq 1 ; x_{m}=1, m>1 .
\end{array}
$$

It should be emphasized that $u_{i, k}(x, t)$ for $m \geq 1$ is governed by the linear equation (2.8) under the linear boundary conditions that come from original problem, which can be easily solved by symbolic computation software such as Mathematica. When $h=-1$, and $H(x, t)=1(2.2)$ becomes

$$
\begin{aligned}
& (1-q) L_{j}\left[\phi_{i}(x, t, q)-u_{i, 0}(x, t)\right]+q N_{j}\left[\phi_{1}(x, t, q), \ldots, \phi_{m}(x, t, q)\right] \\
& \quad=0, i=1, \ldots, m ; j=1, \ldots, n
\end{aligned}
$$

which is used mostly in the homotopy perturbation method [16-20].

For the following numerical examples, we use $L_{j}=\partial / \partial t ; H(x, t)=1$. 


\section{Two-Component Evolutionary System of Order 3: Type I}

In this section, we consider system (1.1)

$$
\begin{gathered}
u_{t}=u_{x x x}+u u_{x}+v v_{x} \\
v_{t}=-2 v_{x x x}-u v_{x}
\end{gathered}
$$

subject to

$$
\begin{aligned}
& u(x, 0)=-6 \operatorname{csch}^{2}\left(\frac{x}{\sqrt{2}}\right) \\
& v(x, 0)=6 \operatorname{csch}\left(\frac{x}{\sqrt{2}}\right) .
\end{aligned}
$$

For application of the homotopy analysis method, we choose the initial approximations as

$$
\begin{aligned}
& u_{0}(x, t)=u(x, 0), \\
& v_{0}(x, t)=v(x, 0) .
\end{aligned}
$$

Employing HAM with the mentioned parameters in Section 2, we have the following zeroorder deformation equations:

$$
\begin{gathered}
(1-q) L_{j}\left[\phi_{1 t}-u(x, 0)\right]=q h\left[\phi_{1 t}-\phi_{1 x x x}-\phi_{1} \phi_{1 x}-\phi_{2} \phi_{2 x}\right] \\
(1-q) L_{j}\left[\phi_{2 t}-v(x, 0)\right]=q h\left[\phi_{2 t}+2 \phi_{2 x x x}+\phi_{1} \phi_{2 x}\right] .
\end{gathered}
$$

Subsequently solving the $N$ th order deformation equations, we obtain

$$
\begin{gathered}
u_{1}(x, t)=3 h t \sqrt{2} \operatorname{csch}^{4}\left(\frac{x}{\sqrt{2}}\right) \sinh (\sqrt{2} x), \\
v_{1}(x, t)=-3 h t \sqrt{2} \operatorname{coth}^{4}\left(\frac{x}{\sqrt{2}}\right) \operatorname{csch}\left(\frac{x}{\sqrt{2}}\right), \\
u_{2}(x, t)=-3 h t \operatorname{csch}^{4}\left(\frac{x}{\sqrt{2}}\right)(2 h t+h t \cosh (\sqrt{2} x)-\sqrt{2}(1+h) \sinh (\sqrt{2} x)), \\
v_{2}(x, t)=\frac{3}{4} h t \operatorname{csch}^{3}\left(\frac{x}{\sqrt{2}}\right)(3 h t+h t \cosh (\sqrt{2} x)-2 \sqrt{2}(1+h) \sinh (\sqrt{2} x)) .
\end{gathered}
$$




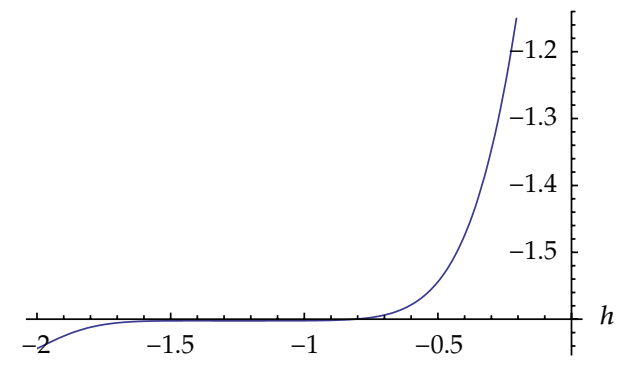

(a)

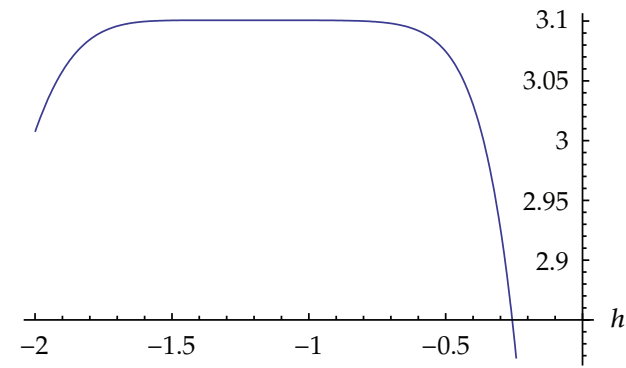

(b)

Figure 1: (a) The $h$ curve of system $(1.1)$ for $u(3,1)$ obtained from the 10th order HAM. (b) The $h$ curve of system $(1.1)$ for $v(3,1)$ obtained from the 10th order HAM.

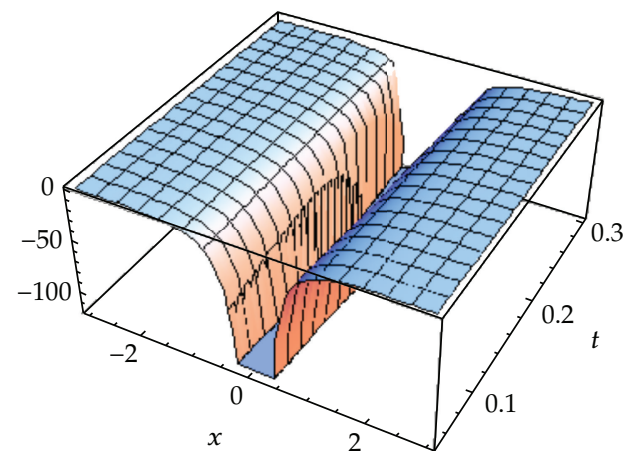

(a)

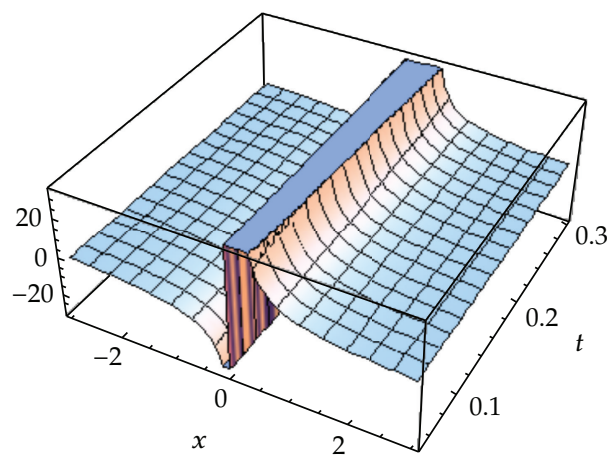

(b)

Figure 2: (a) The obtained $u_{\text {HAM }}$ for system (1.1) using 10-term approximation. (b) The obtained $v_{\text {HAM }}$ for system (1.1) using 10-term approximation. $h=-1.1$.

We use an 10-term approximation and set

$$
\begin{aligned}
& u_{\mathrm{HAM}}(x, t)=\sum_{i=0}^{10} u_{i}(x, t), \\
& v_{\mathrm{HAM}}(x, t)=\sum_{i=0}^{10} v_{i}(x, t) .
\end{aligned}
$$

Comments and illustrations upon the obtained results can be observed in Figures 1 and 2 .

\section{Two-Component Evolutionary System of Order 3: Type II}

In this section, we consider system (1.2)

$$
\begin{gathered}
u_{t}=u_{x x x}+2 v u_{x}+u v_{x}, \\
v_{t}=u u_{x}
\end{gathered}
$$


subject to

$$
\begin{gathered}
u(x, 0)=-\tanh \left(\frac{x}{\sqrt{3}}\right) \\
v(x, 0)=-\frac{1}{6}-\frac{1}{2} \tanh ^{2}\left(\frac{x}{\sqrt{3}}\right) .
\end{gathered}
$$

For application of the homotopy analysis method, we choose the initial approximations as

$$
\begin{aligned}
& u_{0}(x, t)=u(x, 0) \\
& v_{0}(x, t)=v(x, 0) .
\end{aligned}
$$

Employing HAM with mentioned parameters in Section 2, we have the following zero-order deformation equations:

$$
\begin{gathered}
(1-q) L_{j}\left[\phi_{1 t}-u(x, 0)\right]=q h\left[\phi_{1 t}-\phi_{1 x x x}-2 \phi_{2} \phi_{1 x}-\phi_{1} \phi_{2 x}\right], \\
(1-q) L_{j}\left[\phi_{2 t}-v(x, 0)\right]=q h\left[\phi_{2 t}-\phi_{1} \phi_{1 x}\right] .
\end{gathered}
$$

Subsequently solving the $N$ th order deformation equations, we obtain

$$
\begin{gathered}
u_{1}(x, t)=-\frac{h t}{\sqrt{3}} \operatorname{sech}^{2}\left(\frac{x}{\sqrt{3}}\right), \\
v_{1}(x, t)=-\frac{h t}{\sqrt{3}} \operatorname{sech}^{2}\left(\frac{x}{\sqrt{3}}\right) \tanh \left(\frac{x}{\sqrt{3}}\right), \\
u_{2}(x, t)=-\frac{h t}{3} \operatorname{sech}^{3}\left(\frac{x}{\sqrt{3}}\right)\left(\sqrt{3}(1+h) \cosh \left(\frac{x}{\sqrt{3}}\right)-h t \sinh \left(\frac{x}{\sqrt{3}}\right)\right), \\
v_{2}(x, t)=\frac{h t}{6} \operatorname{sech}^{4}\left(\frac{x}{\sqrt{3}}\right)\left(-2 h t+h t \cosh \left(\frac{2 x}{\sqrt{3}}\right)-\sqrt{3}(1+h) \sinh \left(\frac{2 x}{\sqrt{3}}\right)\right),
\end{gathered}
$$

and so on. We use an 10-term approximation and set

$$
\begin{aligned}
& u_{\text {HAM }}(x, t)=\sum_{i=0}^{10} u_{i}(x, t), \\
& v_{\text {HAM }}(x, t)=\sum_{i=0}^{10} v_{i}(x, t) .
\end{aligned}
$$

Comments and illustrations upon the obtained results can be observed in Figures 3 and 4. 


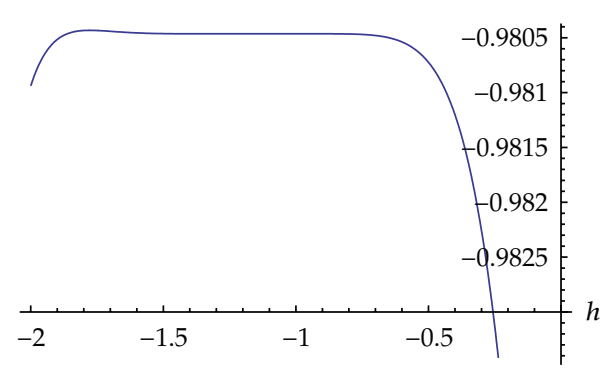

(a)

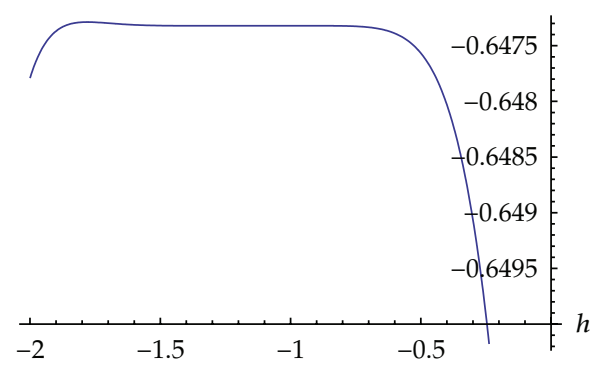

(b)

Figure 3: (a) The $h$ curve of system $(1.2)$ for $u(5,1)$ obtained from the 10th order HAM. (b) The $h$ curve of system $(1.2)$ for $v(5,1)$ obtained from the 10th order HAM.

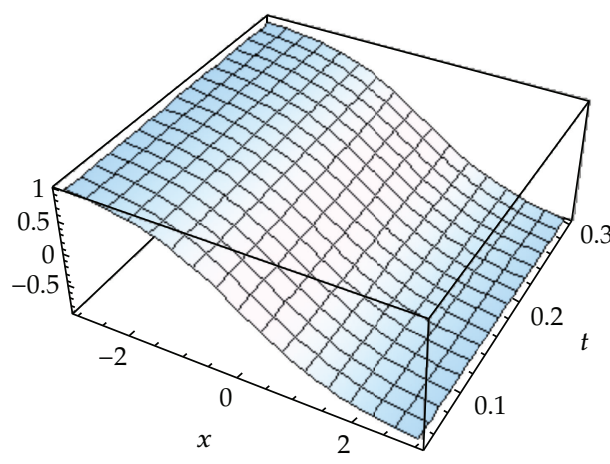

(a)

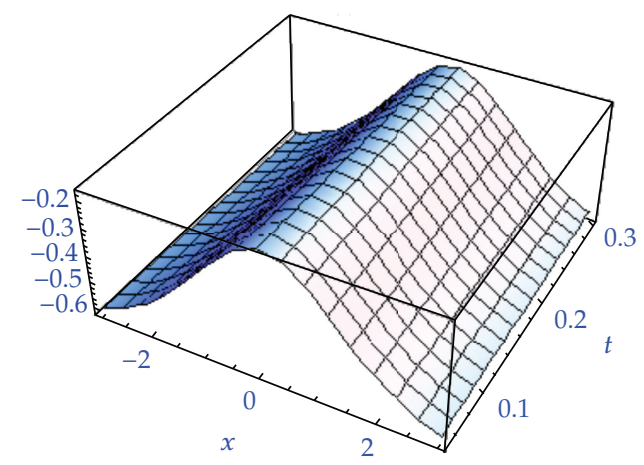

(b)

Figure 4: (a) The obtained $u_{\text {HAM }}$ for system (1.2) using 10-term approximation. (b) The obtained $v_{\text {HAM }}$ for system (1.2) using 10-term approximation. $h=-1.3$.

\section{Two-Component Evolutionary System of Order 2}

In this section, we consider system (1.3)

$$
\begin{gathered}
u_{t}=-3 v_{x x} \\
v_{t}=4 u_{x x}+u^{2}
\end{gathered}
$$

subject to

$$
\begin{aligned}
& u(x, 0)=-\frac{1}{8}-\frac{1}{8} \tan ^{2}\left(\frac{x}{2 \sqrt{3}}\right), \\
& v(x, 0)=-\frac{1}{4 \sqrt{3}} \tan \left(\frac{x}{2 \sqrt{3}}\right) .
\end{aligned}
$$


We proceed in the same manner and choose the initial approximations as

$$
\begin{aligned}
& u_{0}(x, t)=u(x, 0), \\
& v_{0}(x, t)=v(x, 0) .
\end{aligned}
$$

The zero-order deformation equations are

$$
\begin{gathered}
(1-q) L_{j}\left[\phi_{1 t}-u(x, 0)\right]=q h\left[\phi_{1 t}+\phi_{2 x x}\right], \\
(1-q) L_{j}\left[\phi_{2 t}-v(x, 0)\right]=q h\left[\phi_{2 t}-\phi_{1}^{2}-4 \phi_{1 x x}\right] .
\end{gathered}
$$

Subsequently, we obtain

$$
\begin{gathered}
u_{1}(x, t)=\frac{h t}{8 \sqrt{3}} \sec ^{2}\left(\frac{x}{2 \sqrt{3}}\right) \tan \left(\frac{x}{2 \sqrt{3}}\right), \\
v_{1}(x, t)=-\frac{h t}{24} \sec ^{2}\left(\frac{x}{2 \sqrt{3}}\right), \\
u_{2}(x, t)=\frac{h t}{96} \sec ^{4}\left(\frac{x}{2 \sqrt{3}}\right)\left(-2 h t+h t \cos \left(\frac{x}{\sqrt{3}}\right)+2 \sqrt{3}(1+h) \sin \left(\frac{x}{\sqrt{3}}\right)\right), \\
v_{2}(x, t)=\frac{h t}{144} \sec ^{3}\left(\frac{x}{2 \sqrt{3}}\right)\left(-6(1+h) \cos \left(\frac{x}{2 \sqrt{3}}\right)+\sqrt{3} h \sin \left(\frac{x}{2 \sqrt{3}}\right)\right),
\end{gathered}
$$

and so on. We use an 11-term approximation and set

$$
\begin{aligned}
& u_{\mathrm{HAM}}(x, t)=\sum_{i=0}^{10} u_{i}(x, t), \\
& v_{\mathrm{HAM}}(x, t)=\sum_{i=0}^{10} v_{i}(x, t) .
\end{aligned}
$$

The obtained $h$-curve and the HAM solutions of $u(x, t)$ and $v(x, t)$ are given in Figures 5 and 6.

\section{Discussion and Concluding Remarks}

In this paper, we obtained soliton solutions for homogeneous $\mathrm{KdV}$ systems of second and third order by means of homotopy analysis method. The convergence region for the obtained, approximation, is determined by the parameter $h$ as shown in Figures 1, 3, and 5, respectively, for systems ((1.1), (1.2), and (1.3)).

Homotopy analysis method provides us a convenient freely chosen with parameter $h$ in contrast to the Homotopy perturbation method, where $h$ is assumed to be -1 . In this work, $h$ has been chosen to be $-1.1,-1.3$, and -0.7 , respectively, for systems ((1.1), (1.2), 


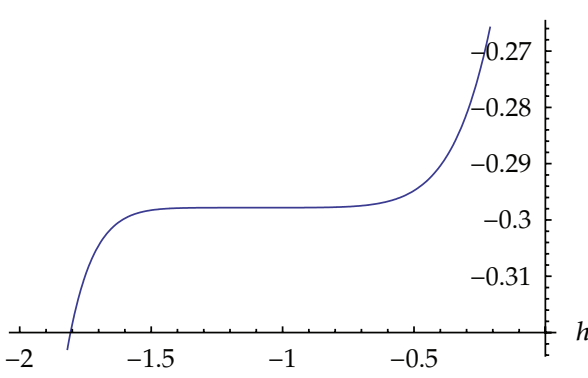

(a)

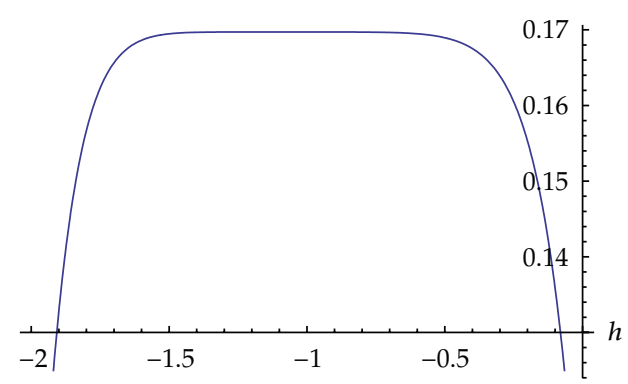

(b)

Figure 5: (a) The $h$ curve of system $(1.3)$ for $u(3,1)$ obtained from the 10th order HAM. (b) The $h$ curve of system $(1.3)$ for $v(3,1)$ obtained from the 10th order HAM.

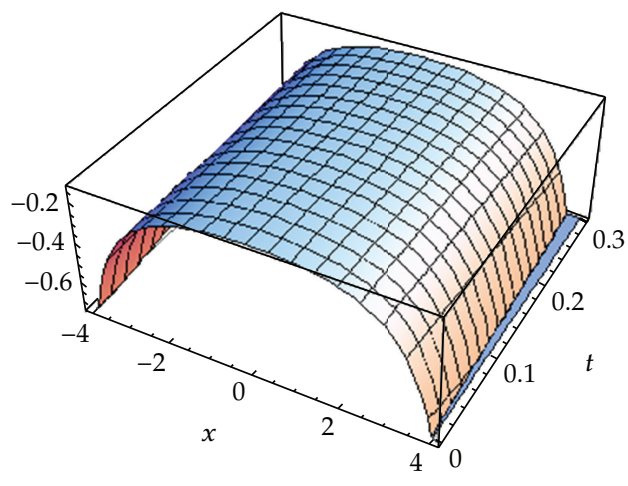

(a)

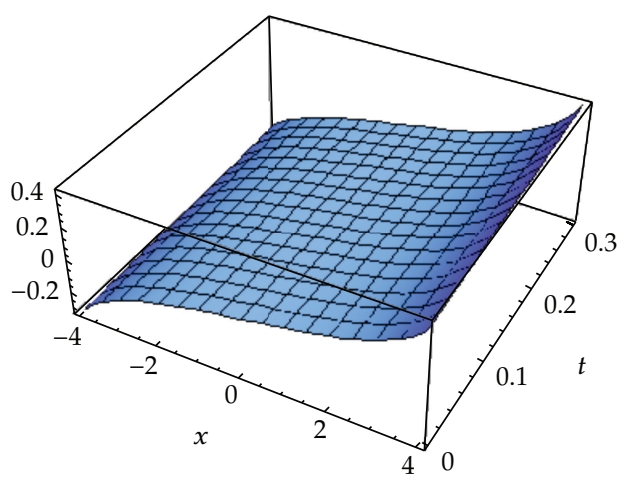

(b)

Figure 6: (a) The obtained $u_{\text {HAM }}$ for system (1.3) using 10-term approximation. (b) The obtained $v_{\text {HAM }}$ for system (1.3) using 10-term approximation. $h=-0.7$.

Table 1: Absolute errors regarding $u(x, t)$ for system (1.1) with $N=10$.

\begin{tabular}{lcccccc}
\hline$x_{i} \mid t_{j}$ & 0.05 & 0.1 & 0.15 & 0.20 & 0.25 & 0.30 \\
\hline-6 & $4.32 \times 10^{-13}$ & $4.09 \times 10^{-12}$ & $2.05 \times 10^{-11}$ & $7.48 \times 10^{-11}$ & $2.24 \times 10^{-10}$ & $5.86 \times 10^{-10}$ \\
-4 & $7.77 \times 10^{-12}$ & $7.78 \times 10^{-11}$ & $4.14 \times 10^{-10}$ & $1.61 \times 10^{-9}$ & $5.16 \times 10^{-9}$ & $1.44 \times 10^{-8}$ \\
-2 & $3.43 \times 10^{-10}$ & $6.30 \times 10^{-9}$ & $5.54 \times 10^{-8}$ & $3.25 \times 10^{-7}$ & $1.46 \times 10^{-6}$ & $5.40 \times 10^{-6}$ \\
2 & $1.25 \times 10^{-12}$ & $1.99 \times 10^{-12}$ & $1.92 \times 10^{-12}$ & $5.76 \times 10^{-12}$ & $1.29 \times 10^{-11}$ & $1.98 \times 10^{-11}$ \\
4 & $9.23 \times 10^{-14}$ & $1.59 \times 10^{-13}$ & $5.30 \times 10^{-14}$ & $4.45 \times 10^{-13}$ & $3.79 \times 10^{-13}$ & $7.06 \times 10^{-13}$ \\
6 & $5.52 \times 10^{-15}$ & $9.60 \times 10^{-15}$ & $2.88 \times 10^{-15}$ & $2.67 \times 10^{-14}$ & $2.02 \times 10^{-14}$ & $4.30 \times 10^{-14}$ \\
\hline
\end{tabular}

Table 2: Absolute errors regarding $v(x, t)$ for system (1.1) with $N=10$.

\begin{tabular}{lcccccc}
\hline$x_{i} \mid t_{j}$ & 0.05 & 0.1 & 0.15 & 0.20 & 0.25 & 0.30 \\
\hline-6 & $2.67 \times 10^{-12}$ & $1.51 \times 10^{-11}$ & $5.27 \times 10^{-11}$ & $1.45 \times 10^{-10}$ & $3.46 \times 10^{-10}$ & $7.47 \times 10^{-10}$ \\
-4 & $1.17 \times 10^{-11}$ & $7.21 \times 10^{-11}$ & $2.81 \times 10^{-10}$ & $8.87 \times 10^{-10}$ & $2.44 \times 10^{-9}$ & $6.15 \times 10^{-9}$ \\
-2 & $1.22 \times 10^{-10}$ & $1.62 \times 10^{-9}$ & $1.21 \times 10^{-8}$ & $6.42 \times 10^{-8}$ & $2.68 \times 10^{-7}$ & $9.44 \times 10^{-7}$ \\
2 & $6.19 \times 10^{-13}$ & $3.18 \times 10^{-12}$ & $5.02 \times 10^{-13}$ & $6.58 \times 10^{-12}$ & $1.03 \times 10^{-11}$ & $2.23 \times 10^{-12}$ \\
4 & $1.61 \times 10^{-13}$ & $7.90 \times 10^{-13}$ & $8.33 \times 10^{-14}$ & $1.39 \times 10^{-12}$ & $1.70 \times 10^{-12}$ & $3.50 \times 10^{-13}$ \\
6 & $3.93 \times 10^{-14}$ & $1.92 \times 10^{-13}$ & $2.42 \times 10^{-14}$ & $3.34 \times 10^{-13}$ & $4.01 \times 10^{-13}$ & $1.00 \times 10^{-13}$ \\
\hline
\end{tabular}


and (1.3)). It is worth noting that the choice of the parameter $h$ in this paper is considered based on the obtained stability region of the $h$-curve for each system; for example, in system (1.1) the stability region of $h$ falls between -1.5 and -0.7 and we considered the midpoint of this interval.

Finally, the absolute errors for the obtained approximate solution of system (1.1) are given in Tables 1 and 2.

\section{References}

[1] S. J. Liao, Proposed homotopy analysis technique for the solution of nonlinear problems [Ph.D. thesis], Shanghai Jiao Tong University, 1992.

[2] S. Abbasbandy, E. Babolian, and M. Ashtiani, "Numerical solution of the generalized Zakharov equation by homotopy analysis method," Communications in Nonlinear Science and Numerical Simulation, vol. 14, no. 12, pp. 4114-4121, 2009.

[3] E. Babolian and J. Saeidian, "Analytic approximate solutions to Burgers, Fisher, Huxley equations and two combined forms of these equations," Communications in Nonlinear Science and Numerical Simulation, vol. 14, no. 5, pp. 1984-1992, 2009.

[4] S. J. Liao, Beyond Perturbation: An Introduction to Homotopy Analysis Method, vol. 2 of CRC Series: Modern Mechanics and Mathematics, Chapman \& Hall/CRC, Boca Raton, Fla, USA, 2004.

[5] A. M. Lyapunov, General Problem on Stability of Motion, Taylor \& Francis, London, UK, 1992.

[6] S. J. Liao, "Notes on the homotopy analysis method: some definitions and theorems," Communications in Nonlinear Science and Numerical Simulation, vol. 14, no. 4, pp. 983-997, 2009.

[7] S. Abbasbandy and A. Shirzadi, "A new application of the homotopy analysis method: solving the Sturm-Liouville problems," Communications in Nonlinear Science and Numerical Simulation, vol. 16, no. 1, pp. 112-126, 2011.

[8] S. Abbasbandy, J. L. López, and R. López-Ruiz, "The homotopy analysis method and the Liénard equation," International Journal of Computer Mathematics, vol. 88, no. 1, pp. 121-134, 2011.

[9] R. C. Aziz, I. Hashim, and S. Abbasbandy, "Effects of thermocapillarity and thermal radiation on flowand heat transfer in a thin liquid film on an unsteady stretching sheet," Mathematical Problems in Engineering, vol. 2012, Article ID 127320, 14 pages, 2012.

[10] A. Bekir, "Applications of the extended tanh method for coupled nonlinear evolution equations," Communications in Nonlinear Science and Numerical Simulation, vol. 13, no. 9, pp. 1748-1757, 2008.

[11] M. Alquran, "Solitons and periodic solutions to nonlinear partial differential equations by the SineCosine method," Applied Mathematics and Information Sciences, vol. 6, no. 1, pp. 85-88, 2012.

[12] M. Alquran, K. Al-Khaled, and H. Ananbeh, "New soliton solutions for systems of nonlinear evolution equations by the rational Sine-Cosine method," Studies in Mathematical Sciences, vol. 3, no. 1, pp. 1-9, 2011.

[13] S. Shukri and K. Al-Khaled, "The extended tanh method for solving systems of nonlinear wave equations," Applied Mathematics and Computation, vol. 217, no. 5, pp. 1997-2006, 2010.

[14] M. Alquran, R. Al-Omary, and Q. Katatbeh, "New explicit solutions for homogeneous Kdv equations of third order by trigonometric and hyperbolic function methods," Applications and Applied Mathematics, vol. 7, no. 1, pp. 211-225, 2012.

[15] A. Jabbari and H. Kheiri, "Homotopy analysis and homotopy Padé methods for $(2+1)$-dimensional Boiti-Leon-Pempinelli system," International Journal of Nonlinear Science, vol. 12, no. 3, pp. 291-297, 2011.

[16] A. M. A. El-Sayed, A. Elsaid, and D. Hammad, "A reliable treatment of homotopy perturbation method for solving the nonlinear Klein-Gordon equation of arbitrary (fractional) orders," Journal of Applied Mathematics, vol. 2012, Article ID 581481, 13 pages, 2012.

[17] M. Alquran and M. Mohammad, "Approximate solutions to system of nonlinear partial differential equations using homotopy perturbation method," International Journal of Nonlinear Science, vol. 12, no. 4, pp. 485-497, 2011.

[18] J. H. He, "Homotopy perturbation technique," Computer Methods in Applied Mechanics and Engineering, vol. 178, no. 3-4, pp. 257-262, 1999.

[19] J. H. He, "A coupling method of a homotopy technique and a perturbation technique for non-linear problems," International Journal of Non-Linear Mechanics, vol. 35, no. 1, pp. 37-43, 2000.

[20] J. H. He, "Homotopy perturbation method: a new nonlinear analytical technique," Applied Mathematics and Computation, vol. 135, no. 1, pp. 73-79, 2003. 


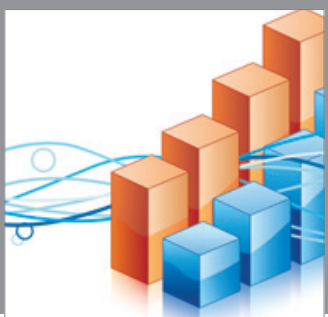

Advances in

Operations Research

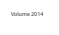

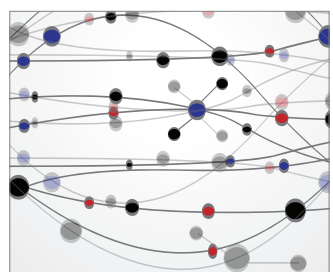

\section{The Scientific} World Journal
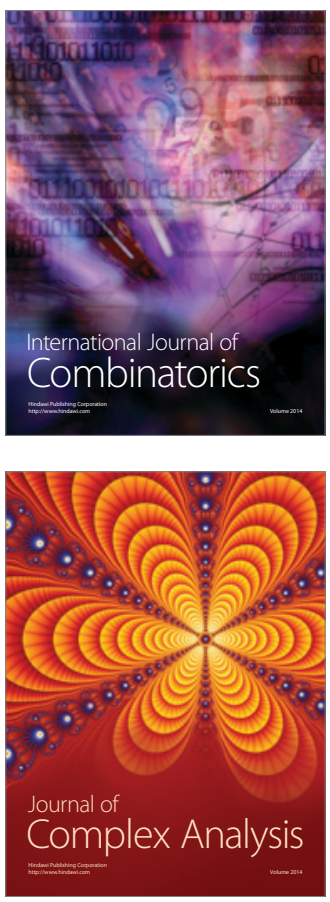

International Journal of

Mathematics and

Mathematical

Sciences
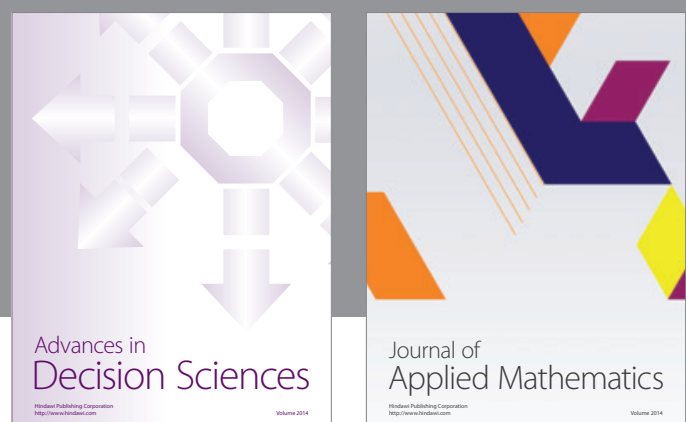

Journal of

Applied Mathematics
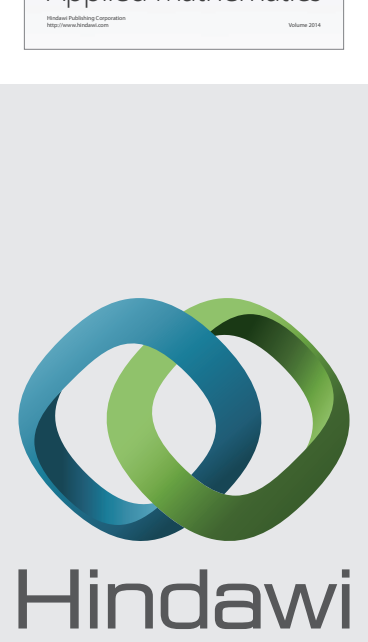

Submit your manuscripts at http://www.hindawi.com
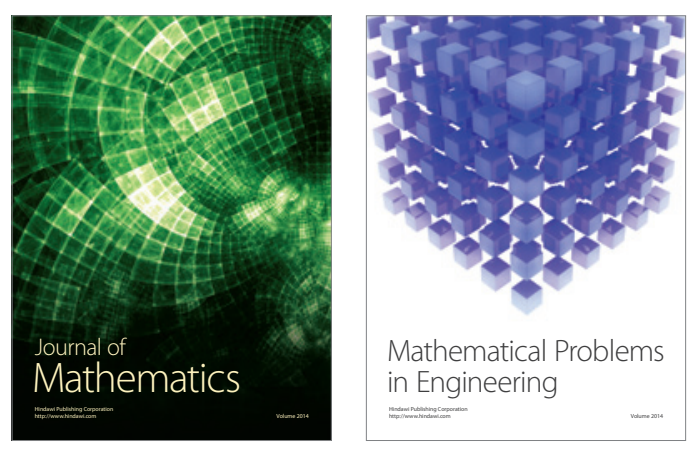

Mathematical Problems in Engineering
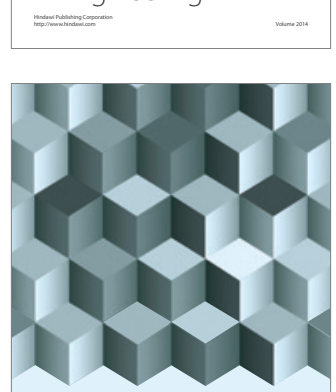

Journal of

Function Spaces
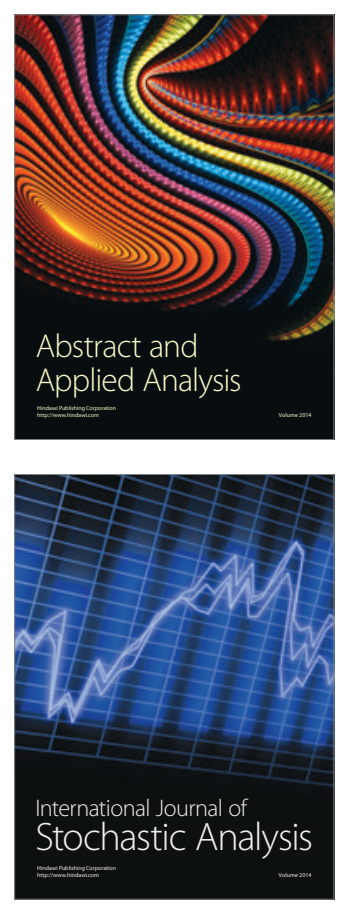

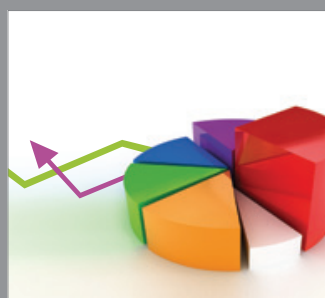

ournal of

Probability and Statistics

Promensencen
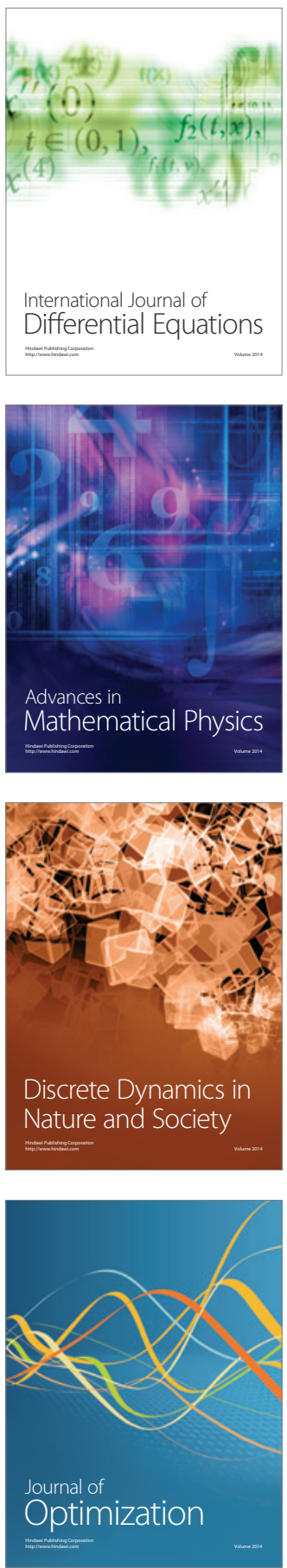